\title{
DEFORMATION MONITORING OF THE SPALLATION NEUTRON SOURCE (SNS)* TUNNELS
}

\author{
J. Error, D. Bruce, J. Fazekas, S. Helus, J. Maines, Oak Ridge National Laboratory (ORNL), U.S.A.
}

\section{Abstract}

The SNS Project is a $1.4 \mathrm{MW}$ accelerator-based neutron source located at Oak Ridge National Laboratory in Oak Ridge, Tennessee. For shielding purposes, 5 meters of native soil backfill has been placed on top of the accelerator tunnel system. This backfill has caused ongoing settlement of the tunnels. The settlement has been monitored by the SNS Survey and Alignment Group in order to determine the patterns of deformation, and to determine when the tunnel system will be stable enough for precise alignment of beam line components. The latest monitoring results indicate that the settlement rate has significantly decreased. This paper introduces the techniques and instrumentation of the monitoring surveys, and describes the types and magnitudes of deformation found.

\section{INTRODUCTION}

The Spallation Neutron Source (SNS) Project was constructed at Oak Ridge National Laboratory (ORNL), on the top of Chestnut Ridge. The initial site grading required the removal of up to 20 meters of soil from the top of the ridge. After construction of the accelerator tunnel system, 5 meters of soil backfill was placed on top of the tunnels to serve as radiation shielding.

The SNS Survey and Alignment Group was concerned about the effect of "recompression settlement" on the stability of the tunnels. That is, the removal of original weight of soil would cause the underlying ground to rise slightly in response. Then the re-application of weight during the backfill stage would result in the recompression of the underlying soil, by $2-5$ centimeters, throughout the tunnel system. These early estimates proved to be well founded.

Monitoring performed on the tunnel floor has shown that the typical magnitude of the recompression settlement has been about 20 millimeters, and that the settlement has not been uniform, even on a local scale. Monitoring surveys have also revealed some horizontal deformation which is probably backfill-related.

The recompression settlement prediction exceeded, by more than two orders of magnitude, the project alignment requirements (typically $0.1 \mathrm{~mm}$ r.m.s.). Therefore the SNS Survey and Alignment Group has been monitoring

* SNS is managed by UT-Battelle, LLC, under contract DE-AC05$00 \mathrm{OR} 22725$ for the U.S. Department of Energy. SNS is a partnership of six national laboratories: Argonne, Brookhaven, Jefferson,

Lawrence Berkeley, Los Alamos and Oak Ridge. the various forms of tunnel deformation, in an effort to determine when the tunnel system will be stable enough for precise alignment of beam line components. The latest monitoring results indicate that the rates of different forms of deformation have significantly decreased, but have not yet dropped to undetectable levels.

\section{MONITORING SURVEYS}

\section{Global Network}

Prior to the construction of the tunnel system, a total of 13 survey monuments were installed across the SNS site. A high-precision 3-D survey network was observed using these monuments, providing a single, unified coordinate system for all of the construction surveyors working on the site. These monuments also provided a stable datum for deformation monitoring surveys. Reobservation surveys have been performed annually on these monuments to check their stability, and to update the coordinates of any monuments disturbed by construction.

\section{Recompression Settlement Monitoring}

For recompression settlement surveys, differential leveling was performed with a Leica NA3003 digital level and bar-coded invar rods. A dense pattern of preciselyrepeatable points was established in the floor of the tunnels, in the form of embedded monument nests. Because of scheduling and manpower restrictions, frequent monitoring of the entire tunnel system was not feasible. Therefore, the Ring to Target Beam Transport (RTBT) tunnel was chosen as a representative section for monthly settlement monitoring. The other portions of the tunnel system were re-observed on an irregular basis. That is, reobservation surveys were performed whenever the magnitude of the settlement required updating monument heights, in order to install or realign beam line components.

\section{Other Deformation Monitoring}

The deformation of the tunnels has not been limited to recompression settlement. Monitoring these other forms of deformation was accomplished by combining measurements from the Leica LTD500 laser tracker and the Leica TDA5005 total station. Monitoring points for these surveys were monument nests located in the floor and on the walls. Again, because of scheduling and manpower restrictions, these surveys were performed on an irregular basis. 


\section{MODES OF DEFORMATION}

\section{Gross Recompression Settlement}

For most of the tunnel system, the backfill operation typically resulted in 20 millimeters of settlement. However, the settlement has exceeded $50 \mathrm{~mm}$ in two separate sections of the tunnel. The greatest settlement has occurred in the downstream portion of the RTBT tunnel, where it has exceeded $180 \mathrm{~mm}$. The wide variation in settlement is due to differences in the nature of the ground beneath the tunnel. The extreme settlement seen in the downstream RTBT tunnel occurs at the point where the tunnel subgrade was over-excavated by 6-7 meters during the construction of the lowest level of the Target Building. This is the location in the tunnel system with the greatest continuing settlement rate: currently $0.5 \mathrm{~mm}$ per month, but decreasing. The SNS project is investigating the use of an automated settlement measuring system in order to continue monitoring for residual settlement in this area while the beam is running.

Recompression settlement was not restricted to the tunnel system alone. Other buildings in close proximity to the backfill have also experienced settlement. This effect was seen in the floor of the Front End Building, where settlement of $5 \mathrm{~mm}$ was seen at the Linac tunnel interface, tapering off linearly for 5 meters into the Front End floor. To a lesser extent, the Target Building also experienced some deformation from the weight of the backfill on the adjacent RTBT tunnel. However, the Target Building was constructed on hundreds of micropiles, so the magnitude of the recompression settlement was limited to $3 \mathrm{~mm}$, and was detectable only immediately adjacent to the RTBT-Target interface. Additionally, the Compressor Building, which was constructed partially on top of the RTBT tunnel fill, has experienced settlement of over $60 \mathrm{~mm}$ in one corner.

\section{Center-to-Side Differential Floor Settlement}

Floor settlement data show that, across the width of the tunnel floor, the sides have been subsiding more than the center. This differential settlement has been found in all parts of the accelerator tunnel system. The magnitude of this effect is relatively small compared to the overall settlement - in a typical tunnel cross-section, the edges of the tunnel floor have settled $5 \mathrm{~mm}$ more than the center. A visible manifestation probably related to this differential settlement is the crack system found running along the centerline of the tunnel floor, parallel to the beam line.

\section{Spreading of Tunnel Floor and Walls}

The floor centerline crack system is also probably related to two other modes of deformation discovered in the tunnel system. Specifically, the tunnel walls have been spreading apart, and the width of the tunnel floor has been increasing. At any selected point in the tunnel system, the magnitude of the floor widening is very similar to that of the wall spreading. Both effects are greater in the wider parts of the tunnel system, such as the
Linac Dump region. Given this proportional nature, one might initially assume thermal expansion as a cause; however, the temperature difference between the monitoring surveys is far too small to account for the magnitude of the change. In the Linac tunnel, which is 4.267 meters $(14 \mathrm{ft})$ wide, the floor has spread by an average of $1.2 \mathrm{~mm}$ since monitoring began in April 2002.

The rate of floor and wall spreading has been decreasing though. A recent deformation study [1] in the Ring tunnel showed that an average of only $0.23 \mathrm{~mm}$ additional spreading occurred between April 2004 and December 2004.

\section{Deformation Associated with Transverse Floor Cracks}

The previously described cracks along the centerline of the tunnel are not the only type of crack found in the tunnel system. Transverse floor cracks, running across the width of the tunnel, also exist. In some parts of the tunnel system, relatively large motions have been detected between adjacent floor monuments separated by such cracks.

This phenomenon was recently studied in detail [1] in the Ring Injection area, because of the presence of two prominent transverse cracks there. In that study, a 50 meter section of the Ring survey network was resurveyed to detect changes in the coordinates of the monuments over a period of eight months. Positional inconsistencies between adjacent monuments separated by transverse cracks were as much as five times larger than otherwise. Two varieties of transverse-crack-related deformation have been identified. The first variety is seen in the differences in recompression settlement experienced by monuments separated by these cracks. The second variety is displacement along the local axis of the tunnel. Specifically, some of the transverse cracks have been widening or narrowing, causing independent motion of the blocks of floor that these cracks separate.

\section{IMPACTS OF DEFORMATION}

Since recompression settlement was expected, steps were taken to minimize the impact on the project. All components were designed with a coarse vertical adjustment range of $5 \mathrm{~cm}$ to allow component installation to continue uninterrupted during backfill, allaying concerns about the settlement displacing components out of their vertical adjustment range.

However, to support accurate component-to-component placement for vacuum connections, repeated network reobservation campaigns were required, as the survey network persistently deformed far beyond component placement tolerances.

Although large cracks (up to $5 \mathrm{~mm}$ in width) have developed in the floors, walls, and ceilings of the tunnels, water leakage is not seen. This is probably due to the placement of a continuous waterproof membrane over the entire tunnel system, prior to the placement of the topmost 0.5 meter of backfill. 


\section{CONCLUSION}

The predictions of recompression settlement due to the backfill operation were accurate. Despite the large initial magnitude of the settlement, the current settlement rates for most of the accelerator tunnel system are detectable only over periods of several months. The largest ongoing settlement, at the downstream end of the RTBT tunnel, has decreased to $0.5 \mathrm{~mm}$ per month, and is still decreasing. The rates of other forms of tunnel deformation, including wall and floor spreading, have also dramatically decreased.

\section{REFERENCES}

[1] SNS Accelerator Systems Division, Survey and Alignment Group, "The State of the SNS Tunnel Survey Control Network: A Sample Deformation Study in the Ring Injection Area," SNS-NOTESRVY-145, Feb. 3, 2005, http://it.sns.ornl.gov/asd/. 